\title{
Retinal Processing Near Absolute Threshold: from Behavior to Mechanism
}

\author{
Greg D. Field*, Alapakkam P. Sampath*, Fred Rieke ${ }^{\dagger}$ \\ Department of Physiology and Biophysics \\ University of Washington \\ Seattle, Washington 98195 \\ email: gfield@u.washington.edu \\ apsampat@u.washington.edu \\ rieke@u.washington.edu \\ telephone: (206) 616-6956 \\ FAX: (205) 685-0619 \\ * these authors contributed equally to this work \\ $\dagger$ to whom correspondence should be addressed
}

To appear in Ann Rev Physiol

Running title: Photon detection in the retina

Keywords: visual sensitivity, photon detection, signal processing, physical limits, scotopic vision.

\section{Abstract}

Vision at absolute threshold is based on signals produced in a tiny fraction of the rod photoreceptors. This requires the rods to signal the absorption of single photons and the resulting signals to be transmitted across the retina and encoded in the activity sent from the retina to the brain. Behavioral and ganglion cell sensitivity has often been interpreted to indicate that these biophysical events occur effectively noiselessly - i.e. that vision reaches limits to sensitivity imposed by the division of light into discrete photons and occasional photon-like noise events generated in the rod photoreceptors. We will argue that this interpretation is not unique and provide a more conservative view of the constraints behavior and ganglion cell experiments impose on phototransduction and retinal processing. We will then summarize what is known about how these constraints are met and identify some of the outstanding open issues.

\section{Contents}

1 Introduction $\quad 2$

2 Behavior 3

2.1 Frequency of seeing experiments . . . . . . . . . . . . . . . . 3

2.2 False positives and internal noise . . . . . . . . . . . . . . . . . . . 4

2.3 Ambiguities in behavioral measurements . . . . . . . . . . . . . . 6

2.4 Summary . . . . . . . . . . . . . . . . . . . . . . 7 
$\begin{array}{lll}3 & \text { Retinal ganglion cells } & 7\end{array}$

4 Phototransduction $r$

5 Comparison of rod noise with behavioral and ganglion cell sensitivity 10

6 Retinal circuitry and interneurons $\quad 10$

6.1 The rod-to-rod bipolar synapse . . . . . . . . . . . . . . . . 12

6.2 The rod bipolar-to-AII amacrine synapse . . . . . . . . . . . . . 16

6.3 The AII-to-cone bipolar and cone bipolar-to-ganglion cell synapses . . . . . . 17

$\begin{array}{lll}7 & \text { Conclusions } & 18\end{array}$

8 Acknowledgments $\quad 19$

\section{Introduction}

Sensory signals are inherently variable. These variations set a fundamental limit to the performance of any system designed to detect and process these signals. In the case of vision, sensitivity cannot exceed the limit set by the quantization of light into discrete photons and the consequent Poisson fluctuations in photon absorption. Several aspects of dark-adapted visual processing approach this limit. Rod photoreceptors reliably signal the absorption of single photons $[1,2]$, and behavioral detection may require absorption of only a few photons [3]. This exquisite sensitivity is crucial for normal night vision. On a moonless night only one rod in 10,000 receives a photon during the integration time of the rod signals (reviewed by [4]). Thus, visually-guided behavior requires the retina to read out and process single photon signals carried by a tiny fraction of the rods while rejecting noise generated in the remaining rods.

Reaching the sensitivity limit imposed by the quantal nature of light requires that intrinsic noise is small compared to that generated by Poisson fluctuations in photon absorption. Both behavioral $[5,6,7]$ and physiological $[8,9,2]$ studies have identified conditions under which this requirement is not met and intrinsic noise limits sensitivity. The intrinsic noise has been associated with spontaneous activation of rhodopsin in the rod photoreceptors $[5,8,9]$. If correct, this idea implies that the retinal readout of single photon responses in the rod array is efficient and effectively noiseless. However, uncertainty in existing estimates of behavioral sensitivity and of rod noise makes this identification tenuous. Several other noise sources - including other sources of rod noise, synaptic noise in retina and cortex, and noise in spike generation - could also contribute to limiting behavioral sensitivity. While it is not clear whether these other noise sources are negligible, behavioral sensitivity does require that they are small.

Behavioral work has motivated studies of how absorbed photons are transduced by rods and how the resulting signals are processed by the retinal circuitry. This work has focused on several questions: (1) How do rods detect single photons [1, 2]?; (2) How are the resulting signals reliably transmitted across retinal synapses $[10,11,12]$ ?; and (3) How are the signals produced by absorption of a few photons coded in the pattern of activity sent from the retina to the brain [8]? Similar problems occur elsewhere in the nervous system. 
For example, signals in some cortical areas, like signals in the rod array, are sparsely coded $[13,14]$. In such cases, appropriate strategies for computations based on the encoded signals must take the sparseness into account. These general questions can be posed clearly in the retina, because the signal and noise properties of the rod inputs can be measured and the stimuli can be precisely controlled.

Here we review what is known about how the mammalian retina works near absolute visual threshold. We begin by discussing evidence from behavioral experiments and recordings from ganglion cells that the visual system can encode the absorption of a small number of photons. We then describe the properties of the noise inferred from behavioral and ganglion cell experiments. A clear prediction from behavioral and ganglion cell sensitivity is that rod photoreceptors can detect single photons; we will summarize the mechanisms responsible and highlight the rod noise sources that could limit sensitivity. Finally we discuss the retinal circuitry that conveys the rod signals to the ganglion cells, emphasizing how this circuitry processes single photon responses.

\section{Behavior}

Behavioral experiments provide estimates of the minimum number of photon absorptions that can be detected and the noise limiting the reliability of detection. These experiments are often interpreted to indicate that humans can detect $<10$ absorbed photons $[3,15,6]$, limited by the spontaneous activation of rhodopsin in the rod photoreceptors [5]. We will argue that these interpretations are not unique. Thus it is currently unclear what process or processes limit behavioral sensitivity. Consequently, although behavior constrains the physiological mechanisms responsible for dark-adapted visual sensitivity, the extent of these constraints is not clear.

\subsection{Frequency of seeing experiments}

Not long after it was established that light is quantized into discrete photons, Lorentz realized that a just-detectable flash delivered about 100 photons to the cornea and hence thought the quantization of light might be of relevance for visual sensitivity (reviewed by [16]). Identifying the minimum number of photons required for seeing from this observation is difficult because of uncertainty in the quantum efficiency - i.e. the fraction of photons at the cornea absorbed by the rods. Estimates of the quantum efficiency based on measurements of the scatter and absorption properties of structures in the eye (the 'absorptive quantum efficiency') range from 0.1-0.3 [17], indicating that 10-30 photons are required for seeing a flash.

The frequency of seeing experiments of Hecht and colleagues [3] and van der Velden [15] estimated the detection threshold and quantum efficiency directly from behavior. Measurement of the fraction of trials in which a flash was seen as a function of the number of photons at the cornea showed a broad transition from flashes that were rarely seen to those frequently seen (e.g. Figure 1A). The threshold and quantum efficiency were estimated based on three assumptions. First, variability in a subject's responses was attributed to the Poisson statistics of photon absorption and the consequent trial-to-trial fluctuations in the number of absorbed photons. Second, only flashes producing at least $\Theta$ absorbed photons were seen. Third, the average number of photons contributing to seeing, $\bar{n}$, was related to the average 
number of photons at the cornea, $\bar{N}$, by an unknown quantum efficiency, $\bar{n}=Q_{E} \bar{N}$. Thus the probability of seeing a flash delivering an average of $\bar{N}$ photons to the cornea is

$$
P_{\text {see }}=\sum_{n \geq \Theta}^{\infty} \frac{\exp \left(-Q_{E} \bar{N}\right)\left(Q_{E} \bar{N}\right)^{n}}{n !} .
$$

This approach estimates the behavioral threshold, $\Theta$, from the steepness of the transition between flashes that are rarely and almost always seen. If $\Theta$ is small, Poisson fluctuations in the number of absorbed photons from one trial to the next will make the transition broad. The transition becomes steeper with increasing $\Theta$.

When the probability of seeing from Equation 1 is plotted against the logarithm of the flash strength, the unknown quantum efficiency shifts the curve along the flash strength axis but does not change its shape. Thus, the quantum efficiency is estimated from the shift required to align Equation 1 with the frequency of seeing data (Figure 1A). From this analysis Hecht and colleagues estimated a threshold of 5-7 photons and a quantum efficiency of $\sim 0.06$. This behavioral quantum efficiency is considerably lower than estimates of the absorptive quantum efficiency, a point we return to below. Even with the higher quantum efficiency, the likelihood that an individual rod absorbed $>1$ photon on any trial is small because the flashes covered an area of the retina containing $\sim 500$ rods. Thus these experiments require that individual rod photoreceptors detect single photons.

\subsection{False positives and internal noise}

The frequency of seeing analysis described above assumes all the noise in the visual system is the result of Poisson fluctuations in photon absorption. If true, visual sensitivity would reach the limit imposed by the division of light into discrete photons. However, observers occasionally report seeing a flash even when none is delivered, and detection threshold depends on the rate of these false positive responses. Barlow demonstrated this dependence by allowing observers to adopt two criteria, answering either 'yes' or 'maybe' in a frequency of seeing experiment; the 'maybe' responses had a lower threshold and higher false positive rate than the 'yes' responses [5] (e.g. Figure 1B). Barlow used these results to argue that the false positives were produced by internal noise that occasionally generated the false perception of a flash. This noise, together with Poisson fluctuations in photon absorption, was interpreted as limiting detection performance.

Sakitt [6] did a more complete version of Barlow's 'yes-maybe' experiment by asking subjects to rate the strength of each of a series of dim flashes on a scale from 0-6, where 0 corresponds to 'did not see anything' and 6 to 'very bright light'. She constructed frequency of seeing curves and estimated thresholds for ratings of 1 or more, 2 or more, 3 or more, etc. This amounts to measuring thresholds with 6 different criteria. The false positive rate and sensitivity decreased as the criterion increased. The experiments of Sakitt and Barlow show that false positives can trade for detection threshold across a wide range of criteria. In this view, different criteria correspond to different signal-to-noise ratios and observers can chose where to operate based on how many mistakes they are allowed to make. These experiments indicate that a small number of photons, perhaps even a single photon, contribute to detection.

Barlow and Sakitt converted the false positive rates in their respective experiments into an estimate of the internal noise limiting performance. They assumed this noise could 

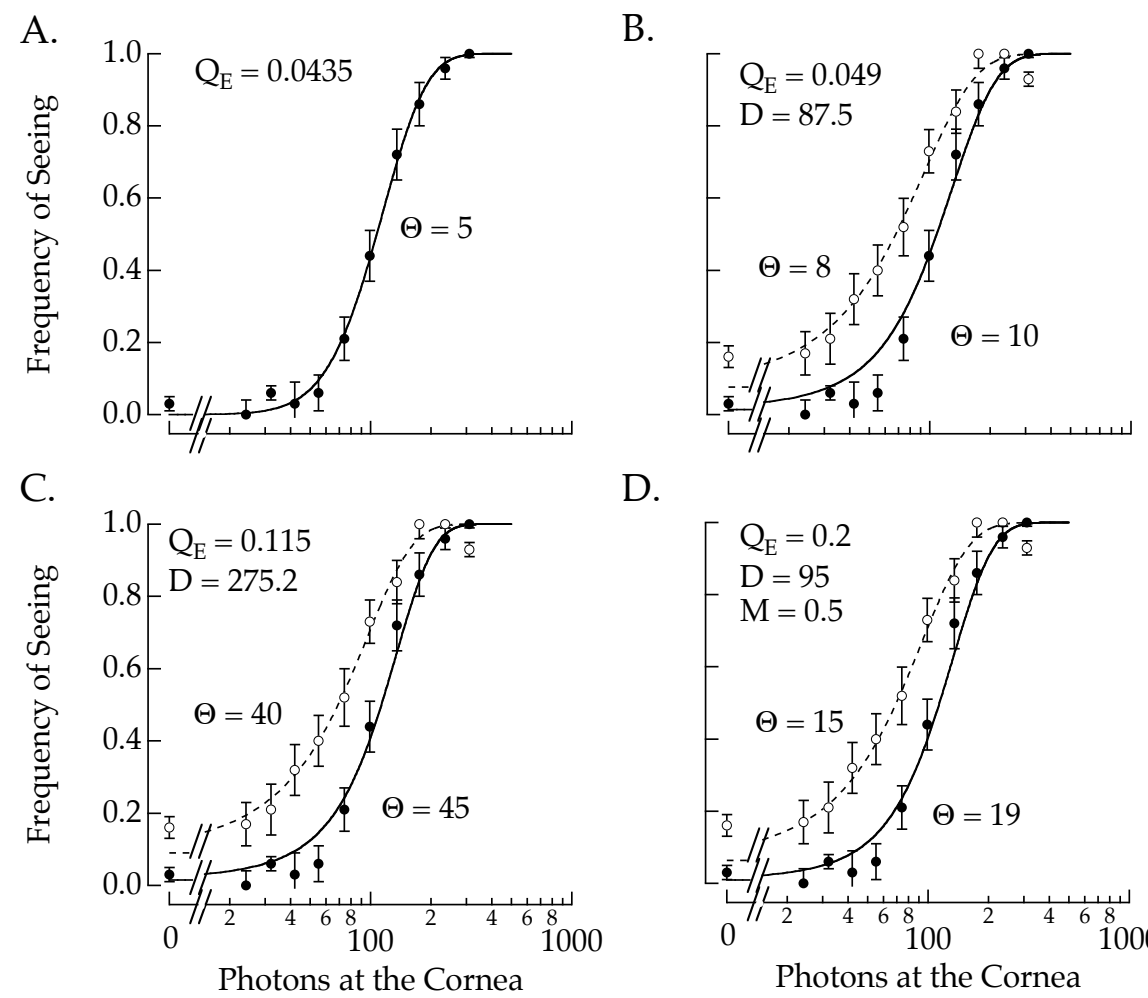

D.

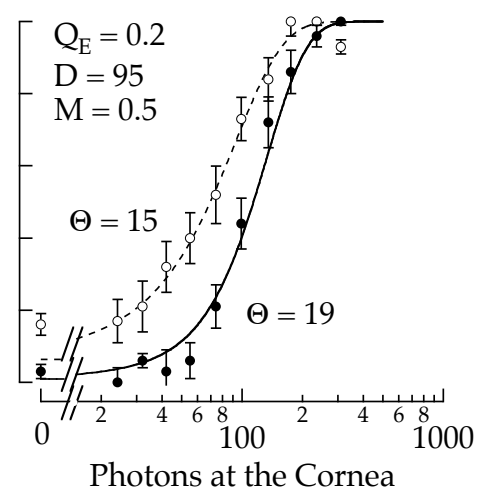

Figure 1: Frequency of seeing data adapted from Teich and colleagues [7]. (A) Data for low false positive rate fit with Equation 1. $\Theta$ is the threshold number of photons, and $Q_{E}$ is the quantum efficiency. (B) Frequency of seeing curves for high (o) and low (•; identical to A) false positive rates from a single subject. Fits from Equation 2 include an additive Poisson noise, constrained to be equal for each curve, and different thresholds. (C) Data in $\mathrm{B}$ replotted and fit with Equation 2 with a higher quantum efficiency, higher thresholds and increased additive Poisson noise. (D) Data replotted and fit with Equation 3, which includes multiplicative Poisson noise. The multiplicative noise allows for a higher quantum efficiency, a lower threshold, and less additive Poisson noise.

be expressed as an additive 'dark light' complete with Poisson fluctuations. In this case, following Equation 1, the probability of seeing is

$$
P_{\text {see }}=\sum_{n \geq \Theta}^{\infty} \frac{\exp \left(-Q_{E}(\bar{N}+D)\right)\left(Q_{E}(\bar{N}+D)\right)^{n}}{n !} .
$$

Here $D$ is the additive Poisson noise (the dark light), expressed as an equivalent number of photons at the cornea. In darkness (i.e. $\bar{N}=0$ ) the probability of seeing is nonzero because of the dark light. Barlow fit the 'yes' and 'maybe' results with Equation 2 assuming they shared a common amount of dark light but had different thresholds (e.g. Figure 1B). Sakitt similarly fit her data such that only the threshold changed between different criteria. Estimated values of the dark light, and difficulties in obtaining a unique estimate, are discussed below. 


\subsection{Ambiguities in behavioral measurements}

The central problem with interpretation of the behavioral experiments summarized above is that the fits to the frequency of seeing curves are not unique. We have described the discrepancy between the behavioral and absorptive quantum efficiencies: behavioral measurements place the quantum efficiency between 0.03-0.06, while direct estimates based on losses within the eye range from 0.1-0.3. Barlow suggested that this discrepancy could originate because the behavioral quantum efficiency can trade for additive Poisson noise when fitting frequency of seeing data with Equation 2 [5]. This is illustrated in Figure 1C, which shows fits to the frequency of seeing data from Teich and colleagues [7] with a quantum efficiency of 0.11. Raising the quantum efficiency increased the threshold by a factor of $\sim 4.5$ and the dark light by a factor of $\sim 3$ when compared to fits with a quantum efficiency of 0.05 (Figure 1B).

Uncertainty in the quantum efficiency produces a nearly 10-fold range in estimates of threshold and dark light. Expressed as an equivalent rate of photon-like noise events in each rod photoreceptor (using estimates of the spatial and temporal summation of the rod array, the rod density, and an assumed quantum efficiency), the dark light ranges from 0.002 to $0.03 \mathrm{sec}^{-1}[18]$. This large range of values makes it impossible to draw a strong association between the dark light and noise originating in the rods.

Figures 1B-C illustrate two examples in a range of possible fits to the frequency of seeing data. At one end of this continuum, the number of photons required for detection is $<10$, the dark light is close to estimates of rod noise, but the behavioral quantum efficiency is very low compared to the absorptive quantum efficiency. This suggests that at least half of the photons absorbed by the rods are not processed. Although it sounds counterintuitive, this can be an effective processing strategy as we discuss below. At the other end of the continuum, the behavioral and absorptive quantum efficiencies agree, but the threshold number of photons and the amount of dark light are high. These extremes provide qualitatively different views of retinal processing. In the first, the retina efficiently and noiselessly processes the rod signals. In the second, the dark light cannot be explained by rod noise alone and instead post-rod processing must be noisy or inefficient.

Another possible resolution of the discrepancy between behavioral and absorptive quantum efficiencies is that the additive noise model is wrong. Lillywhite [19] showed that multiplicative noise could help resolve this discrepancy. Such noise could arise if several Poisson noise sources operate sequentially, and hence the product of their probability distributions determines the response statistics. Additive noise is still required to explain the false positives. With both additive and multiplicative Poisson noise, the probability of seeing becomes

$$
P_{\text {see }}=\sum_{n \geq 0}^{\infty} \frac{\exp \left(-Q_{E}(\bar{N}+D)\right)\left(Q_{E}(\bar{N}+D)\right)^{n}}{n !} \sum_{s \geq \Theta}^{\infty} \frac{\exp (-M n)(M n)^{s}}{s !}
$$

The term inside the first sum is the probability of $n$ photon-like events given the quantum efficiency $Q_{E}$, the mean number of photons at the cornea $\bar{N}$, and the dark light $D$. The second sum determines the probability that the response $s$ is equal to or greater than the threshold $\Theta$ given $n$ absorbed photons. $M$ is the gain from $n$ to $s$, such as might be observed if one photon generated two spikes on average in a ganglion cell. Figure 1D shows fits for the frequency of seeing data according to Equation 3. A combination of additive and multiplicative noise allows for a quantum efficiency of 0.2 and relatively low thresholds [20]. 


\subsection{Summary}

Behavioral measurements of dark-adapted sensitivity place several constraints on visual processing: (1) individual rods can detect single photons; (2) false positives and sensitivity can trade for one another; and (3) signals from a small number of photons, perhaps a single photon, influence perception. A key open issue is whether the retina is efficiently processing the rod signals: Can the dark light be attributed to noise events in the rods? Does the apparent discrepancy between behavioral and absorptive quantum efficiencies result from the discarding of photon responses and/or the presence of multiplicative noise? Alternatively, is the discrepancy a result of incorrectly assuming the threshold number of photons and the amount of dark light are relatively small? The physiological work described below provides some answers.

\section{$3 \quad$ Retinal ganglion cells}

The elegance and the curse of the behavioral experiments is that they lump together all factors that could lower sensitivity, including many central factors [17, 21]. These central factors are a nuisance if the aim is to constrain retinal processing. An alternative is to focus on the fidelity of signals in the retinal ganglion cells. The most extensive of such studies are in the anesthetized cat.

Barlow, Levick and colleagues [22, 8, 23] recorded extracellular spikes from individual ON ganglion cells (probably ON-X according to [9]) in darkness and in response to dim light flashes. The spontaneous activity in darkness consisted of bursts of several spikes. The distribution of intervals between bursts was approximately exponential, indicating that the bursts occur largely independently. This behavior is consistent with the idea that the maintained discharge is produced by additive Poisson noise consisting of independent, discrete noise events, each of which produces a burst of $\sim 3$ spikes.

Barlow and colleagues also analyzed responses to flashes delivering 5-50 photons at the cornea using a frequency of seeing analysis like that used in Sakitt's behavioral measurements [8] and receiver operator characteristic analysis [23]. They concluded that the performance of dark-adapted ganglion cells could be explained with the following assumptions: (1) the quantum efficiency was 0.18 ; (2) each absorbed photon caused the ganglion cell to generate 2-3 extra spikes; and (3) detection was limited by both Poisson fluctuations in photon absorption and additive Poisson noise arising from discrete noise events (dark light) occurring at a rate of $5-6 \mathrm{sec}^{-1}$.

Mastronarde provided additional evidence for Barlow's observations by recording simultaneously from pairs of cat retinal ganglion cells [9]. He found that nearby cells exhibited correlated bursts of 2-3 spikes in darkness and that weak backgrounds increased the rate of these correlated bursts. Cross-correlation functions calculated between the spike trains of two nearby cells had a slow component with a time scale of $\sim 50 \mathrm{msec}$; this relatively long time scale is consistent with a common input to the cell pair. Pairs of on cells and pairs of OFF cells were positively correlated and ON-OFF pairs were anticorrelated. These correlations included on and ofF center X ( $\beta$ or midget-like) and Y ( $\alpha$ or parasol-like) ganglion cells.

Based on the similarity of the correlated bursts in darkness and in the presence of a dim background, Mastronarde argued that the slow correlations resulted from photon-like events in the rods - either due to spontaneous or light-activated rhodopsin. Assuming a 
quantum efficiency of 0.12 and an approximate receptive field size, Mastronarde estimated that each photon produced 1.5-2 spikes, similar to the 2-3 spikes per burst estimated from autocorrelation functions computed from the cell's spike trains in darkness. Noise bursts occurred at a rate of $2-6 \mathrm{sec}^{-1}$. A rate of $6 \mathrm{sec}^{-1}$ corresponds to $\sim 0.002$ events $/ \mathrm{rod} / \mathrm{sec}$ given the rod density [24] and receptive field size [25, 26]. These conclusions are in agreement with those of Barlow and colleagues [8].

Mastronarde's and Barlow's results are consistent with the idea that the retina can detect and process single photon responses. However, as with the behavioral data, this is not a unique interpretation. More conservatively, their results argue for a source of discrete, independent noise events originating in the rods or in the retinal circuitry. This noise likely originates in the AII amacrine cells or earlier (see Retinal circuitry and interneurons section) to explain the anticorrelation between ON and OFF cells. We provide additional arguments in the next section that the identification of the ganglion cell bursts with rod noise is tenuous.

Several studies provide evidence for non-additive noise originating downstream of the rods. Frishman and Levine [27] recorded from ganglion cells in the presence of a steady dim background light, or a modulated light with the same mean intensity. They showed that the statistics of ganglion cell spiking under these two conditions were inconsistent with models with only photoreceptor noise. In addition, Lillywhite [19] and Saleh and Teich [28] argue that multiplicative noise can account for many aspects of the ganglion cell signals previously attributed to additive noise. Multiplicative noise can explain the observation that the variance of the ganglion cell spike count is greater than the mean [28]. As with the behavioral data, multiplicative noise may help explain why the inferred quantum efficiency of $\sim 0.15$ is lower than the absorptive quantum efficiency of $\sim 0.3$ in cat $[17,19,7]$. If each photon produces more than one spike, however, multiplicative noise will play a lesser role in limiting sensitivity than Poisson fluctuations in photon absorption. Furthermore, multiplicative noise alone cannot explain the ganglion cell's spontaneous spiking activity in darkness.

\section{Phototransduction}

The first prediction from behavioral and ganglion cell experiments - that rods can detect single photons — was confirmed more than 25 years ago by Baylor and colleagues [1, 2]. These studies identified three important functional properties of the rod's single photon responses (e.g. Figure 2A): (1) the electrical response triggered by photon absorption is highly amplified; (2) the rod maintains low noise in darkness; and (3) individual single photon responses show low trial-to-trial variability. Combined efforts in biochemistry, molecular biology and physiology have since explored the molecular basis of each of these properties (reviewed in [29]).

The activity of a single rhodopsin molecule is amplified in several stages (reviewed in [30]). First, rhodopsin itself catalyzes the activation of hundreds or thousands of copies of the G-protein transducin [31, 32]. Each transducin activates a single phosphodiesterase (efficiency $\sim 0.8$ ), and each phosphodiesterase hydrolyzes many cGMP molecules. This results in the closure of cGMP-gated channels and a 1-2 pA reduction in inward current [2]. In total, activation of a single rhodopsin leads to the degredation of $10^{5}-10^{6}$ cGMP molecules and the failure of $\sim 10^{6} \mathrm{Na}^{+}$ions to flow into the outer segment. This scheme applies to all vertebrate rods, however the fidelity of the single photon response varies considerably from 


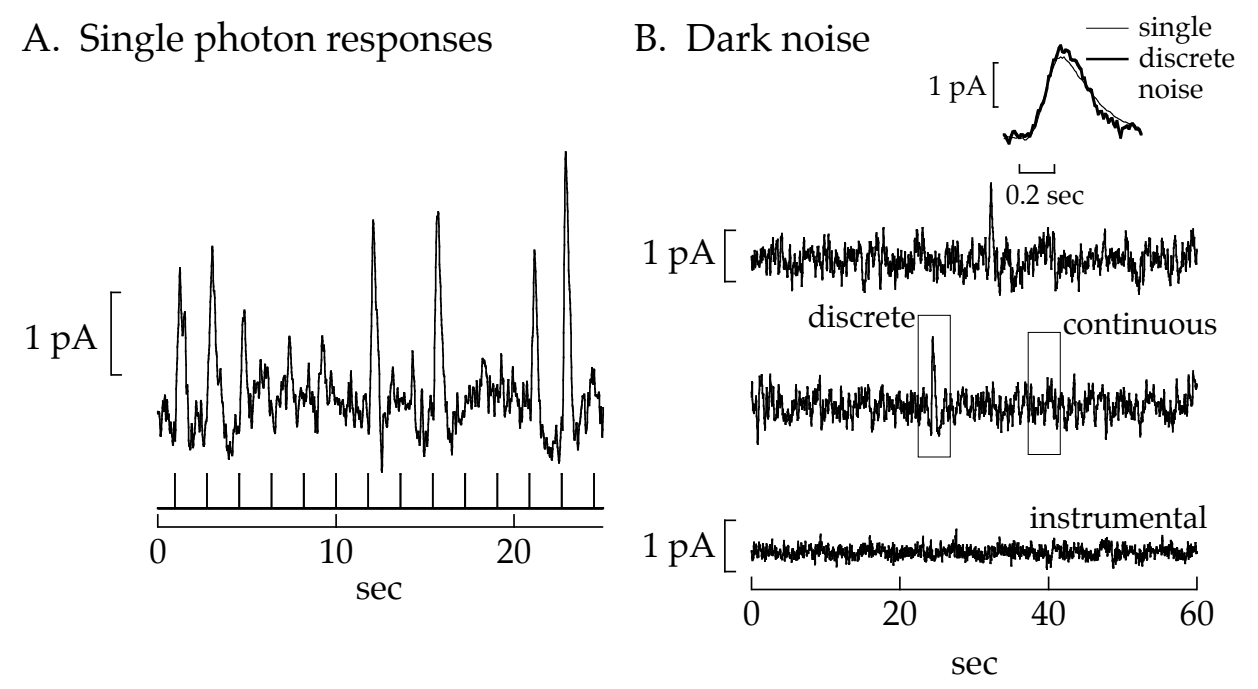

Figure 2: Single photon responses and dark noise in a primate rod. (A) Responses to a repeated dim flash producing an average of $0.5 \mathrm{Rh}^{*}$. (B) Two sections of dark record showing discrete and continuous dark noise. The average of 8 discrete noise events is compared to the cell's average single photon response in the inset. A section of record in saturating light to eliminate the transduction current and isolate instrumental noise is shown below.

one species to another. For instance, the signal-to-noise ratio of the single photon response is $\sim 6$ in primate rods [2,33] and $\sim 3$ in mouse rods [34].

Dark noise in the rod signals comes from spontaneous activation of rhodopsin and phosphodiesterase [35, 2, 36]. Spontaneous rhodopsin activation produces discrete noise events indistinguishable from single photon responses (Figure 2B); these occur once every 200-400 sec in a monkey rod [2] (GDF and FR unpublished). Thus each of the rod's $\sim 10^{8}$ rhodopsin molecules activates spontaneously every 500-1000 years on average. Spontaneous phosphodiesterase activation produces continuous fluctuations in the rod current with a magnitude about one fourth the size of the single photon response (Figure 2B). These current fluctuations have a similar frequency composition to the rod's single photon response, and thus occasional large continuous noise deviations will mimic single photon responses [2].

In addition to the low dark noise of the phototransduction cascade, the rod's single photon responses vary little from one to the next, particularly when compared to the variations expected for signals initiated by a single molecule $[37,38,33]$. This low variability poses an interesting molecular design question: How is the activity of a single rhodopsin molecule regulated to avoid the expected statistical variations in its lifetime? Low variability is also required if the visual system is to count photons, as suggested by Sakitt's behavioral results, and may enable the rod responses to encode precisely the time of photon arrival [37]. 


\section{Comparison of rod noise with behavioral and gan- glion cell sensitivity}

Behavioral measurements and recordings from ganglion cells provide an estimate of the intrinsic noise that limits absolute sensitivity. This noise has usually been associated with spontaneous activation of rhodopsin, which produces additive Poisson noise [5, 8, 9]. Indeed, the rate of photon-like noise events in monkey rods is in approximate agreement with estimates of the noise limiting human behavior (reviewed by [18]). Furthermore, the noise limiting the sensitivity of cat ganglion cells appears to consist of discrete bursts of spikes similar to those produced by dim backgrounds [8, 9].

Several issues make the association of the dark light with photon-like noise events in the rods tenuous. First, fits to the frequency of seeing curves depend on several factors (quantum efficiency, additive Poisson noise, and multiplicative Poisson noise) that can trade for one another (Figure 1). This precludes a unique interpretation. Second, in the cat ganglion cell experiments, the number of rods providing input to the recorded cells was not determined and the properties of noise in cat rods have not been measured. Experiments in toad provide additional evidence that behavior approaches limits set by spontaneous rhodopsin activation. In particular, the temperature dependence of behavioral threshold is correlated with the temperature dependence of the rate of photon-like noise events in the rods [39]. However, the effect of temperature on behavior is very different in frogs [40]. This difference among closely related species, along with differences in both the signal-to-noise ratio of the single photon responses and the underlying retinal circuitry in amphibian and mammalian retina, makes it impossible to generalize from toads to humans.

What other sources of noise might limit sensitivity? First, continuous noise in mammalian rods can generate large fluctuations that look like true photon responses [2, 34]. Second, noise downstream of the rods, e.g. synaptic noise or noise in spike generation, could contribute to either additive or multiplicative noise. Determining the identity of the noise limiting ganglion cell sensitivity remains a key question in how the retina works at low light levels. If the noise can be entirely attributed to the rods, the retinal readout of the rod array must be efficient and effectively noiseless. This is a strong constraint.

\section{Retinal circuitry and interneurons}

Behavioral, ganglion cell, and rod experiments illustrate that single photons are detected at the initial stage of retinal processing and suggest that single photon responses reliably traverse the retina. Understanding the retinal mechanisms responsible for this sensitivity and the identity of the noise limiting sensitivity will require following the rod's single photon responses across the retina. Our current understanding of how single photon responses are processed by retinal interneurons is primitive compared to our knowledge about rod and ganglion cell physiology.

Rod signals are transmitted across the mammalian retina through at least three pathways: the rod bipolar pathway, the rod-cone pathway and the rod-ofF pathway (Figure 3; see also $[41,42])$. The rod bipolar pathway, which is a special feature of mammalian retina, has long been considered the primary route for rod signals at light levels near absolute threshold. Only recently has a solid experimental basis for this idea been developed [43, 44, 45]. In 


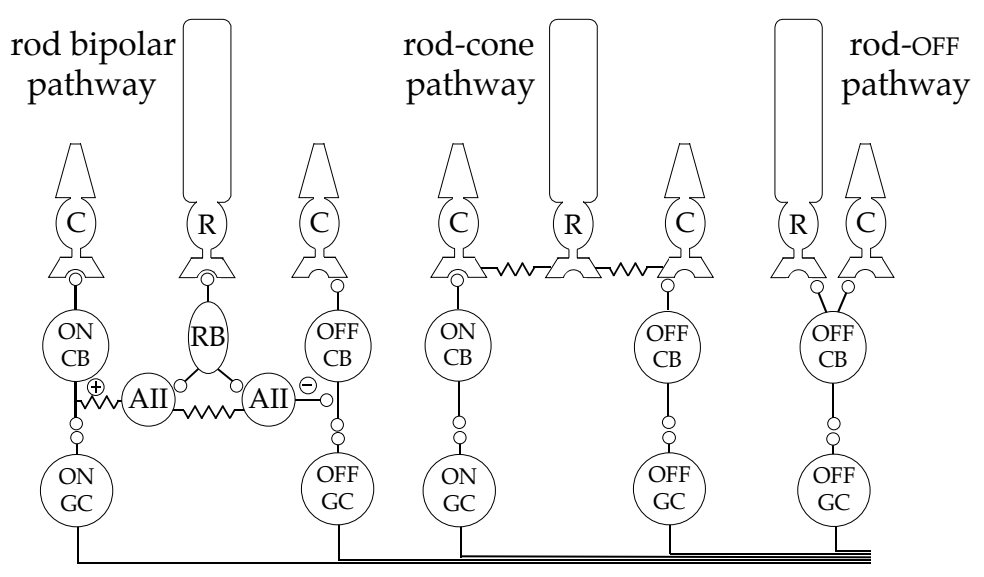

Figure 3: Pathways for rod signals. In mammalian retina responses generated by rod photoreceptors $(\mathrm{R})$ can reach ganglion cells $(\mathrm{GC})$ by three routes. In the rod bipolar pathway [46, 47, 48], rod signals are sent to rod bipolar cells (an ON -type bipolar) (RB), which subsequently send these signals to a network of electrically-coupled AII amacrine cells. AII amacrines are electrically coupled to on cone bipolar (CB) cells, and connected by a glycinergic synapse to OFF cone bipolar cells. These cone bipolars send signals to on and ofF ganglion cells. In the rod-cone pathway [49, 24, 50], rods are electrically coupled to cones (C), which relay both ON and OFF signals to ganglion cells through cone bipolars (CB). In the rod-OFF pathway $[43,51,52]$, ganglion cells receive rod input through ofF cone bipolar cells, which make synaptic contacts with rods.

particular, isolation of the pathways through a combination of genetic and pharmacological manipulations indicates that the rod bipolar pathway operates at light levels at least 10-fold lower than the others. Thus behavior at light levels near absolute threshold is mediated by the rod bipolar pathway.

Rod signals in the rod bipolar pathway are passed to rod bipolar cells, a type of on or depolarizing bipolar cell that receives exclusive rod input [46, 47, 48]. Rod bipolars differ from prototypical bipolar cells because they make few or no direct contacts with ganglion cells. Instead, each rod bipolar contacts several electrically coupled AII amacrine cells [48]. AII amacrine cells contact ON-cone bipolar cells through gap junctions, and OFF-cone bipolar cells through sign-inverting glycinergic synapses. Cone bipolar cells transmit rod signals from the rod bipolar pathway to ganglion cells. Below we describe what is known about how each component of this pathway processes single photon responses and identify some of the outstanding open questions. A more detailed account of the anatomy and general function can be found in Bloomfield and Dacheux [42]. 


\subsection{The rod-to-rod bipolar synapse}

Work on transmission of single photon responses from rods to rod bipolar cells has emphasized three issues: (1) the presynaptic mechanisms permitting reliable transmission of the small voltage changes produced by photon absorption; (2) separation of the rod's single photon responses from continuous noise; and (3) a speeding of the single photon response in bipolar cells compared to rods.

Behavioral sensitivity requires that rods generate electrical responses to single photons and that some of these responses are transmitted to rod bipolar cells. The presynaptic hyperpolarization produced by absorption of a photon is only $1-2 \mathrm{mV}$ in amplitude [50]. The small size of the presynaptic signal makes transmission challenging compared to a central synapse where the voltage changes are 100 times larger. Rods are depolarized in darkness and continuously release glutamate at a specialized ribbon-type synapse [53, 54]. Photon absorption hyperpolarizes the rod and produces a reduction or pause in release. Random pauses or slowing due to statistical fluctuations in release will masquerade as true photon events, and thus produce a source of noise potentially limiting visual sensitivity [10]. Such a noise source could account for some of the dark light discussed in the Behavior and Ganglion cell sections. The vesicle release rate and statistics determine the magnitude of this synaptic noise.

Salamander rods release vesicles at a rate of at least $400 \mathrm{sec}^{-1}$ at the dark potential of $-40 \mathrm{mV}$ as indicated by capacitance measurements [55]; these rods, however, have larger active zones and more release sites than mammalian rods. Rao-Mirotznik and colleagues [12] argued that mammalian rods must maintain a dark release rate of at least $80-100 \mathrm{sec}^{-1}$ to insure that random pauses in release produce false photon-like events at a rate lower than the rate of spontaneous rhodopsin activation in the rod outer segment (taken as $0.01 \mathrm{sec}^{-1}$ ). Their argument is based on two assumptions: (1) vesicle release obeys Poisson statistics; and (2) release is completely abolished for $\sim 100$ msec during the single photon response.

The first assumption of Rao-Mirotznik [12] is supported by work on synapses made by spiking cells. In particular, the release of vesicles at the neuromuscular junction follows Poisson statistics as long as the release probability at each fusion site is low [56]; however, these are synapses that can reload between the brief bouts of exocytosis produced by action potentials. The statistics of vesicle release could be different when the synaptic machinery is forced to operate continuously as for rods in darkness; each of the $\sim 40$ fusion sites [11] in a mammalian rod spherule would have to release several vesicles per second to support a rate of $100 \mathrm{sec}^{-1}$. Variability in vesicle release could be reduced if each vesicle fusion site exhibited a refractory period following release, just as a refractory period can reduce variability in spike generation $[57,58]$. Indeed, tracking of single vesicles in goldfish bipolar terminals (also a ribbon-type synapse) indicates that they are not available for exocytosis for $\sim 0.1 \mathrm{sec}$ after arrival at the membrane [59]. If present, a similar refractory period at release sites in the rod terminal could reduce the variance in the number of vesicles released, thus reducing the required mean rate.

The second assumption made by Rao-Mirotznik and colleagues [12], that release is completely suppressed by the single photon response, has not been directly tested. This is because the small size of mammalian rods makes direct measurement of the voltage dependence of transmitter release difficult. This relation has been studied in amphibians by recording simultaneously from rods and postsynaptic cells [60,61], by monitoring capacitance changes produced by exocytosis [62], and by detecting released glutamate photometrically [63]. These 
A. $\operatorname{Rod}$

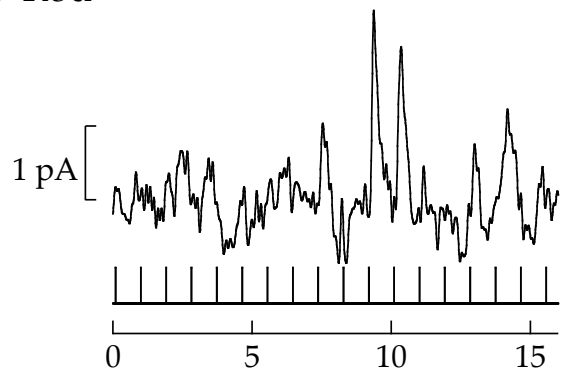

B. Rod bipolar

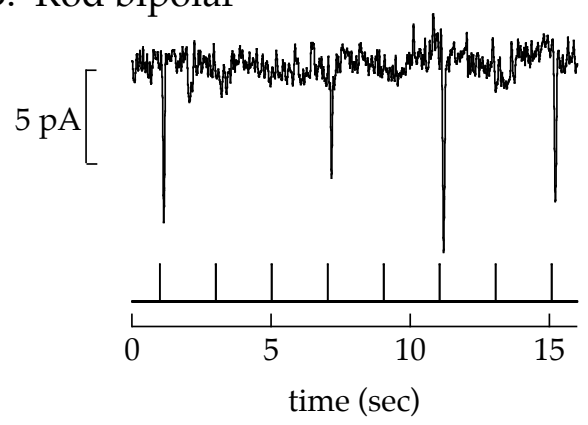

C.

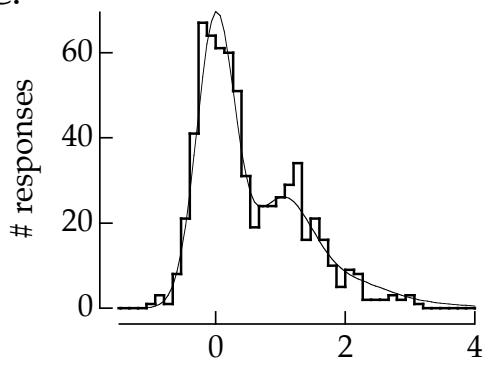

D.

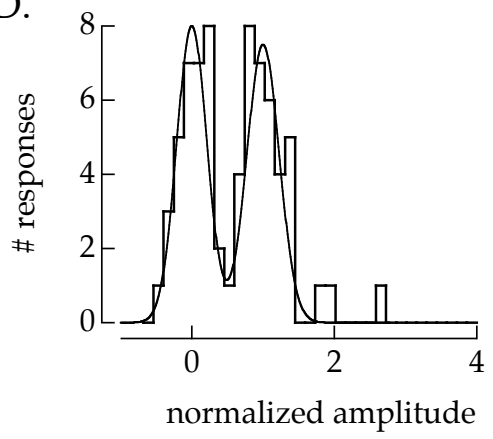

Figure 4: Rod bipolar single photon responses are more discrete than rod single photon responses. (A) Rod photocurrent and (B) rod bipolar current recorded while delivering periodic flashes that generate $\sim 0.6$ activated rhodopsins per rod. Rod currents were measured with a suction electrode. Rod bipolar currents were measured under voltage clamp with the cell held at $-60 \mathrm{mV}$. Histograms of the normalized response amplitudes are plotted in (C) rods and (D) rod bipolar cells. Each histogram has been fit with a sum of weighted Gaussian functions. Adapted from Field and Rieke [34].

studies indicate that the release rate changes exponentially for a $2-5 \mathrm{mV}$ voltage change. A similar voltage dependence in mammalian rods would cause the $1-2 \mathrm{mV}$ single photon response to suppress the rate of vesicle fusion by at most $2 / 3$. Keeping synaptic noise below rod outer segment noise in this case would require a dark release rate exceeding $400 \mathrm{sec}^{-1}$. Direct measurement of the statistics of vesicle release and the consequences of the resulting synaptic noise remains a key experiment.

A second issue at the rod-to-rod bipolar synapse is the separation of single photon responses from continuous noise. The continuous noise appears relatively innocuous in recordings from single rods (e.g. Figure 2); however, when signals from multiple rods are combined at light levels near absolute threshold, noise generated in all the rods threatens to overwhelm the light responses generated in a few rods. Linear summation of the rod inputs is a poor strategy under these conditions because each rod input contributes to the sum without regard to the likelihood that the rod absorbed a photon. A more effective strategy is to first pass the rod inputs through a thresholding nonlinearity that retains signals from those rods 

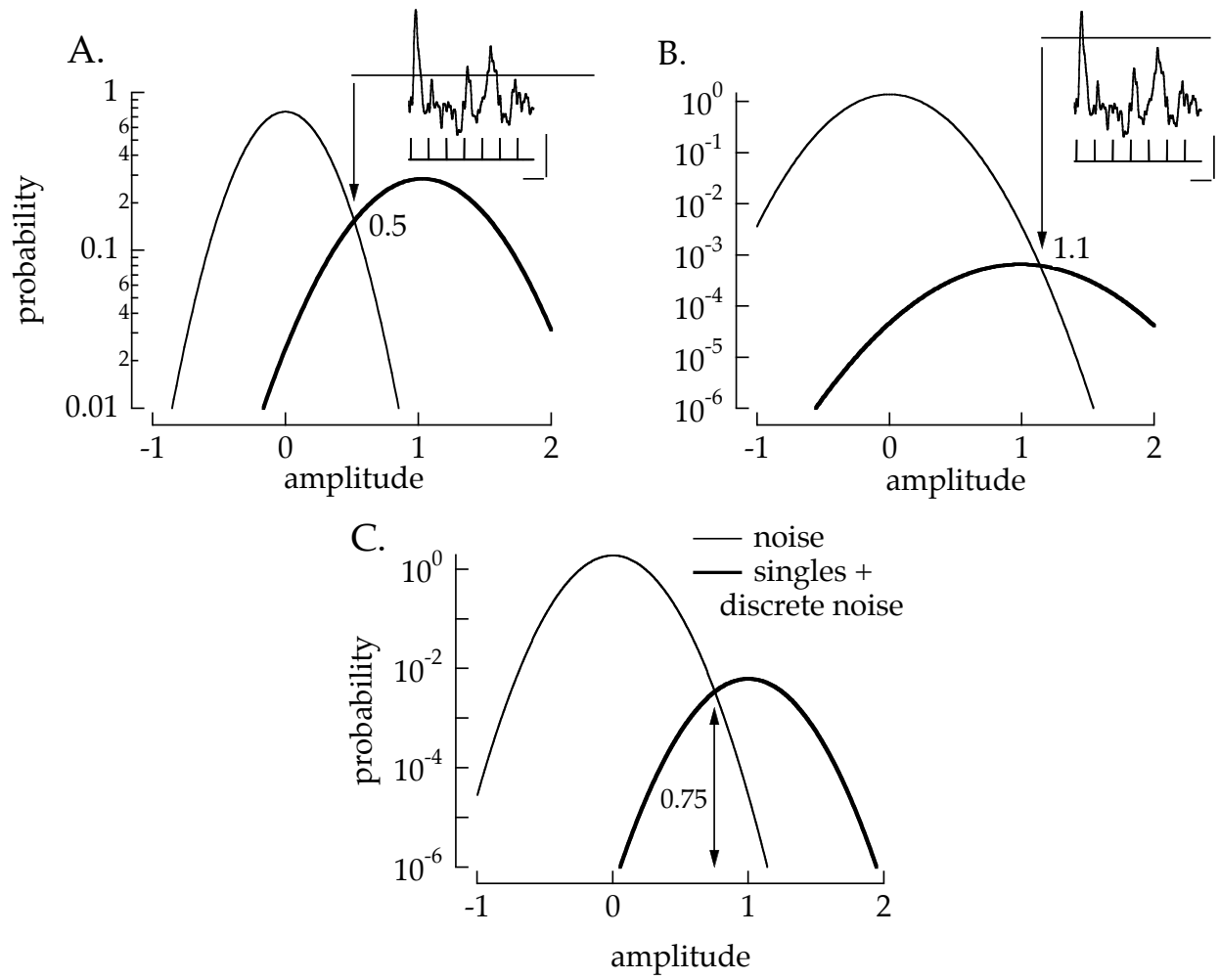

Figure 5: Optimal separation of signal and noise by a thresholding nonlinearity. (A) Comparison between the distribution of the continuous dark noise (thin line) and the distribution of signals generated by single photon responses plus the spontaneous activation of rhodopsin (thick line) for a rod photoreceptor at a light level of $0.6 \mathrm{Rh}^{*}$. The crossing point of the distributions (0.5) indicates the position of the optimal threshold. Insets shows the position of such a threshold relative to the rod responses. (B) Same as (A) but at a light level of $0.0001 \mathrm{Rh}^{*}$ (near absolute threshold). The optimal position of the threshold moves to a higher value as the light level is decreased. (C) Same comparison as (B) for a rod bipolar cell at visual threshold. The separation of signal and noise in rod bipolar cells (see Figure 5) lowers the position of the optimal threshold (0.75).

generating single photon responses and rejects signals from those rods generating noise $[2,64]$ (see Figure 4). Convergence of 20-100 rods onto a rod bipolar makes the rod-to-rod bipolar synapse the final opportunity to implement such a threshold. Indeed, Field and Rieke [34] found that such a nonlinearity exists at the rod-to-rod bipolar synapse. As a consequence, the single photon responses of mouse rod bipolar cells are much more identifiable than those of the rods (Figure 4).

While convergence dictates the need for a thresholding nonlinearity at the rod-to-rod bipolar synapse, it does not predict where the threshold should be positioned relative to the single photon response. Instead, the optimal position of the threshold is set by the light level and properties of the rod noise (Figure 5A-B). The light level at visual threshold can be thought of as a prior probability of 0.0001 that an individual rod generates a single 
A. Salamander

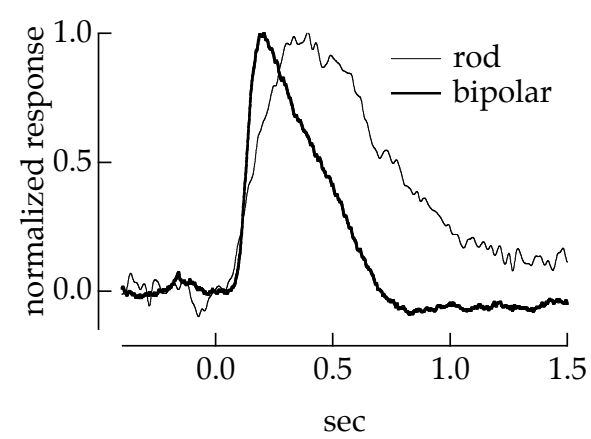

B. Mouse

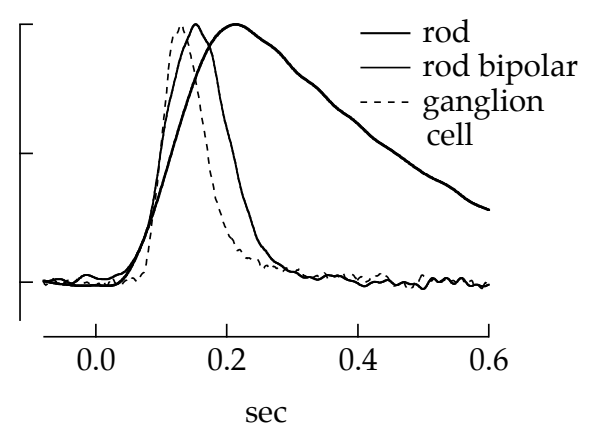

Figure 6: (A) Normalized dim flash responses from a salamander rod and bipolar cell recorded simultaneously [68]. (B) Normalized dim flash responses from a mouse rod, rod bipolar cell, and ON ganglion cell synaptic current.

photon response. Photon-like noise events increase this probability to $\sim 0.0007$ (for an 0.1 sec integration time) since they cannot be distinguished from real responses (these noise events were neglected in Field and Rieke [34]). Because of this prior, all signals smaller than the average single photon response in a mouse rod are more likely noise than signal, and should be rejected. Thus only relatively large single photon responses should be retained - i.e. those to the right of the crossing point between the signal and noise distributions in Figure 5B. The observed threshold is close to this optimal position [34]. Rejection of single photon responses sounds like a poor processing strategy, however the resulting decrease in noise dramatically improves the fidelity of the rod signals. Rejection of some single photon responses may contribute to the low quantum efficiencies inferred from behavior and ganglion cell recordings when compared to the absorptive quantum efficiency.

Electroretinograms (ERG) provide additional evidence for a nonlinearity in signal transfer from rods to rod bipolars [65, 66]. The inferred position of the threshold from ERG recordings is lower than that found in slice recordings, suggesting that few photon responses are discarded at the rod-to-rod bipolar synapse. The position inferred from ERG measurements implies one of the following: (1) the threshold is not well placed to separate signal and noise; (2) the component of the ERG attributed to the rod bipolar cells does not effectively isolate events occurring at the rod-to-rod bipolar synapse; or (3) that existing rod noise measurements $[35,2,36,67]$ do not accurately describe rod noise in vivo. Because the ERG does not measure rod noise, resolving this issue requires measuring signal and noise from single cells under conditions close to those in vivo.

Sampath and Rieke [69] found that the thresholding nonlinearity at the rod-to-rod bipolar synapse was produced by saturation of the G-protein cascade in the rod bipolar dendrites. Glutamate from the rods activates metabotropic receptors on the rod bipolar dendrites, which leads to closure of non-selective cation channels through a poorly understood signaling cascade [70]. Reduction in glutamate release during the rod light response reduces receptor activity, opens channels, and leads to depolarization of the rod bipolar cell. In darkness, glutamate release in the rod is sufficient to saturate a component of the rod bipolar transduction cascade and cause the bipolar current to be insensitive to small changes in rod 
voltage and the corresponding changes in glutamate release. Larger changes, such as those produced during the single photon response, relieve this saturation and produce an electrical response in the rod bipolar cell.

The ability of a threshold to separate single photon responses from noise requires that signals are not mixed between neighboring rods prior to reaching the rod synaptic terminal. This is clearly not the case in amphibians, where rods are strongly electrically coupled $[71,72,73,74]$. Gap junctions are also present between rods in some rodent retinas [52] but have not been described in cat or primate. The discreteness of the single photon responses in the rod bipolar currents, and sensitivity inferred from behavior and ganglion cells, suggests that gap junctions between rods are not functional near absolute threshold [24].

A third aspect of signal transfer from rods to bipolar cells is the speeding of the response in the bipolar cells compared to the rods [75, 65, 34] (Figure 6). The response speeding indicates a reduced synaptic gain at low temporal frequencies, or high-pass filtering. Indeed, paired recordings in amphibian retina indicate that presynaptic mechanisms cause synaptic transmission between rods and bipolars to preferentially transmit temporal frequencies near $2 \mathrm{~Hz}$ [68]. This filtering is well suited for the task of identifying the times of photon arrival based on the rod currents [76, 77], as temporal frequencies below $1 \mathrm{~Hz}$ carry little information about photon arrival time and temporal frequencies above $4 \mathrm{~Hz}$ are dominated by noise. This qualitative argument can be formalized to predict the kinetics of the bipolar response based solely on the rod signal and noise. In mouse, the kinetics of rod-mediated responses in retinal ganglion cells are only slightly sped compared to those in the rod bipolars (Figure 6); thus the time course of the rod signals is dominated by rod phototransduction and the rod-to-rod bipolar synapse.

The work summarized in this section identifies the rod-to-rod bipolar synapse as a key component of both transmission and processing of the rod responses. Not only must this synapse maintain low noise, but linear and nonlinear mechanisms serve to speed the rod single photon responses and separate them from continuous noise produced in rod phototransduction. Processing at the rod-to-rod bipolar synapse is well matched to the signal and noise properties of the rod signals.

\subsection{The rod bipolar-to-AII amacrine synapse}

Rod bipolar cells make ribbon synapses onto several types of amacrine cells [46], but have not been observed to contact ganglion cells directly. The connections to the AII and A17 amacrine cells are the best studied. The A17 amacrines (S1/S2 amacrines in rabbit retina) modulate the rod signals by providing inhibitory feedback to rod bipolar synaptic terminals $[78,79,80,81,82]$. Although the AII amacrines are a requisite component of the rod bipolar pathway, little is known about how single photon responses traverse the rod bipolar-to-AII amacrine synapse. Below we focus on open questions at the rod bipolar-to-AII amacrine synapse, in particular how the pattern of convergence and divergence affects photon detection.

AII amacrine cells in cat receive converging input from 20-30 rod bipolar cells, each of which gets input from $\sim 15$ rods [48]. Thus the AII amacrine cell gets direct input from $\sim$ 300-450 rods. At visual threshold, a small fraction $(<0.01)$ of the converging rod bipolar inputs to an AII amacrine carry a light-driven signal. Thus the AII amacrine, like the rod bipolar, faces the problem of convergence of sparse, noisy inputs. Convergence implies that 
a thresholding nonlinearity like that operating at the rod-to-rod bipolar synapse could serve to retain selectively rod bipolar single photon responses while rejecting noise [83]. As is the case at the rod-to-rod bipolar synapse, the best position of such a threshold is dictated by the light level and signal and noise of the rod bipolar cells, independent of convergence.

Noise in the rod bipolar inputs consists of fluctuations in the rod bipolar electrical signals (Figure 5C) and noise produced by stochastic fluctuations in vesicle fusion at the synapse. These noise sources, particularly the synaptic noise, have not been characterized sufficiently to determine how severely linear summation by the AII amacrine would compromise sensitivity. Figure $5 \mathrm{C}$ gives a rough estimate of the distributions of photon-like events and noise in the rod bipolar cell at absolute threshold assuming synaptic noise is negligible. The separation of the signal and noise distributions is greater than that in the rod responses (Figure 5B-C); thus an optimally positioned threshold at the rod bipolar-to-AII synapse (arrow, Figure $5 \mathrm{C}$ ) would reject a smaller fraction of the single photon responses than are rejected at the rod-to-rod bipolar synapse. Figure 5C suggests that at absolute visual threshold the smallest $10-15 \%$ of the rod bipolar single photon responses would have a greater probability of being noise than signal and should be discarded. This nonlinear summation would improve the signal-to-noise ratio of the AII signals by a factor of $\sim 20$ compared to linear summation. There is at present no evidence for or against a threshold at the rod bipolar-to-AII amacrine synapse.

Divergence causes the signal from a single rod to be represented in several AII amacrine cells. A single rod typically sends outputs to 2 rod bipolar cells, and these two rod bipolar cells contact $\sim 5$ AII amacrine cells [48]. This divergence will correlate light-dependent signals in nearby AIIs. Noise generated at the rod bipolar-to-AII synapse should be independent in different AII amacrines, however, and thus the fidelity of the rod signal may be improved by averaging across AIIs $[83,84]$. Such averaging is provided by electrical coupling between nearby AII amacrines [85, 86, 87], which causes the signals to spread from one AII amacrine to $\sim 20$ neighbors under dark-adapted conditions [88]. AII amacrine cells, unlike rods and rod bipolar cells, can also generate $\mathrm{Na}^{+}$action potentials [89]. Modeling work suggests that the resulting nonlinear electrical properties may amplify single photon responses more than noise in a collection of electrically coupled AIIs [83]. The role of these action potentials in signal transmission at visual threshold has not been determined experimentally.

\subsection{The AII-to-cone bipolar and cone bipolar-to-ganglion cell synapses}

Single photon responses in the AII amacrine cell network are passed to ON and OFF cone bipolar cells through distinct mechanisms. ON signals from AII amacrine cells pass through gap junctions to on cone bipolar cell terminals. Electrical signaling through these gap junctions preferentially transmits signals from AII amacrines to cone bipolars due to the large difference $(\sim 5$-fold) in input impedance of the AII amacrines and the cone bipolar cells [87]. Signals from AII amacrines are passed to OFF cone bipolar cell terminals through inhibitory glycinergic synapses [90]. The properties of glycinergic signal transmission from AII amacrines to OFF cone bipolar cells are not well understood. Connections to ON and OFF bipolars do not appear to be entirely promiscuous, because some ON and OFF ganglion cells receive exclusive cone input [91, 44, 45], requiring bipolar cells that do not get rod input. This also suggests that some cones do not make functional gap junctions with rods (see Figure 3). 
Cone bipolar cells convey rod signals directly to ganglion cells. Because all rod signals are conveyed to ganglion cells through cone bipolar cells, no ganglion cells should receive exclusive rod input. Although the cone bipolar-to-ganglion cell synapse has not been studied at light levels near absolute threshold, a good deal is known about how these synapses operate at cone light levels (reviewed by [92]).

\section{Conclusions}

We have reviewed what is currently known about retinal processing near absolute visual threshold. Behavioral experiments $[3,6,7]$ and in vivo retinal ganglion cell recordings [8, 9] predict that rod photoreceptors can detect single photons and indicate a source(s) of additive Poisson noise that can be expressed as an equivalent input light or dark light [2]. Some of the dark light clearly originates from spontaneous activation of rhodopsin in the rods. However, attributing all of the dark light to this source causes the behavioral and absorptive quantum efficiencies to differ substantially. Imposing that the quantum efficiencies are similar requires a large increase in the dark light, making it unlikely that rod noise alone limits sensitivity. These provide two very different views of retinal processing: the first implies the rod signals are efficiently and noiselessly read out by the retinal circuitry, the other implies that a significant amount of additive noise is introduced post-photoreceptor.

The comparison of the noise limiting behavior with noise due to spontaneous activation of rhodopsin neglects at least three issues. First, such a comparison fails to consider other sources of noise in the rods, particularly continuous noise $[35,36]$. Single photon responses can be separated from continuous noise by a threshold-like nonlinearity at the rod-rod bipolar synapse $[2,64]$. Such a threshold, however, would also reject a substantial fraction of the rod's single photon responses [34], and thus contribute to limiting sensitivity. This may be one of the reasons that the absorptive and behavioral quantum efficiencies differ. Second, the assumption that the intrinsic noise can be modeled as an additive Poisson source may be wrong. A combination of additive and multiplicative noise [19, 7] can explain behavioral and ganglion cell sensitivity while providing closer agreement of behavioral and absorptive quantum efficiencies. Third, there is more to rod vision than detecting the presence or absence of dim lights. For example, estimating motion relies on extracting temporal information from the rod array. The noise limiting such computations may differ from that limiting detection. Reproducibility of the rod's single photon responses may permit photon arrival times to be encoded precisely [37], and bandpass filtering at the rod synapse may be a first step in extracting this temporal information [76, 68].

To date, the main success story in our understanding of the retinal basis of photon detection has been work establishing how rod photoreceptors detect single photons. The same sort of quantitative and mechanistic description is lacking in our understanding of how the retina reads out the rod signals. We are beginning to understand how the first synapse in the retina may be optimized for processing retinal signals, but even here significant gaps in our knowledge remain. In particular, we know neither the statistics of vesicle release, nor how the rod signals are being sped. Our knowledge of how single photon responses are processed at subsequent synapses and encoded in the electrical activity of downstream retinal interneurons is even more incomplete. In addition, recent anatomical and physiological work has identified 'alternative' pathways that rod signals can take through the mammalian retina. 
The function of these additional pathways is not clear, although a possible explanation is that they serve to process the rod signals over a wide range of light levels [41].

Vision is one of several examples in neurophysiology where sensory performance is exquisitely sensitivite (reviewed by [93]). Pheromone detection in insects may approach the single molecule limit [94]. Hearing approaches sensitivity limits set by thermal motion of the auditory hair cell stereocilia [93]. Electroreceptors can detect voltage gradients of $\sim 10 \mathrm{nV} / \mathrm{cm}$ [95], much smaller than voltage changes required to open most ion channels. These examples of extreme sensitivity challenge our understanding of sensory transduction, processing, and encoding. Studies of photon detection in the retina provide an opportunity to understand how the underlying biophysical mechanisms meet these challenges.

\section{Acknowledgments}

We thank Horace Barlow, E.J. Chichilnisky, Thuy Doan, Felice Dunn, and Valerie Uzzell for valuable comments on the manuscript and W. Rowland Taylor and Robert Smith for pointing out the importance of photon-like noise events in the analysis of Figure 5. Support was provided by the NIH through grant EY-11850 (FR) and NRSA EY-14784 (APS).

\section{References}

[1] DA Baylor, TD Lamb, and K-W Yau. Responses of retinal rods to single photons. $J$ Physiol, 288:613-634, 1979.

[2] DA Baylor, BJ Nunn, and JL Schnapf. The photocurrent, noise and spectral sensitivity of rods of the monkey macaca fascicularis. J Physiol, 357:575-607, 1984.

[3] S Hecht, S Shlaer, and MH Pirenne. Energy, quanta, and vision. J. Gen. Physiol., 25:819-840, 1942.

[4] J. Walraven, C. Enroth-Cugell, DC. Hood, MacLoed DIA., and JL Schnapf. The control of visual sensitivity, pages 53-101. Academic Press, 1990.

[5] HB Barlow. Retinal noise and absolute threshold. J Opt Soc Am, 46(8):634-639, 1956.

[6] B Sakitt. Counting every quantum. J Physiol, 223(1):131-150, 1972.

[7] MC Teich, PR Prucnal, G Vannucci, ME Breton, and WJ McGill. Multiplication noise in the human visual system at threshold. 3. the role of non-poisson quantum fluctuations. Biol Cybern, 44(3):157-165, 1982.

[8] HB Barlow, WR Levick, and M Yoon. Responses to single quanta of light in retinal ganglion cells of the cat. Vision Res, Suppl 3:87-101, 1971.

[9] DN Mastronarde. Correlated firing of cat retinal ganglion cells. ii. responses of x- and y-cells to single quantal events. J Neurophysiol, 49(2):325-349, 1983.

[10] G Falk and P Fatt. Physical changes induced by light in the rod outer segment of vertebrates. The Handbook of Sensory Physiology, Volume VII/1, 1972. 
[11] R Rao, G Buchsbaum, and P Sterling. Rate of quantal transmitter release at the mammalian rod synapse. Biophys J, 67(1):57-63, 1994.

[12] R Rao-Mirotznik, G Buchsbaum, and P Sterling. Transmitter concentration at a threedimensional synapse. J Neurophysiol, 80(6):3163-3172, 1998.

[13] MP Young and S Yamane. Sparse population coding of faces in the inferotemporal cortex. Science, 256(5061):1327-1331, 1992.

[14] WE Vinje and JL Gallant. Sparse coding and decorrelation in primary visual cortex during natural vision. Science, 287(5456):1273-1276, 2000.

[15] HA van der Velden. The number of quanta necessary for the perception of light in the human eye. Opthalmologica, 111:321-331, 1946.

[16] MA Bouman. History and present status of quantum theory in vision. Sensory Communication, 1961.

[17] HB Barlow. Retinal and central factors in human vision limited by noise. Vertebrate Photoreception, pages 337-351, 1977.

[18] K Donner. Noise and the absolute thresholds of cone and rod vision. Vision Res, 32(5):853-866, 1992.

[19] PG Lillywhite. Multiplicative intrinsic noise and the limits to visual performance. Vision Res, 21(2):291-296, 1981.

[20] MC Teich, PR Prucnal, G Vannucci, ME Breton, and WJ McGill. Multiplication noise in the human visual system at threshold: 1. quantum fluctuations and minimum detectable energy. J Opt Soc Am, 72(4):419-431, 1982.

[21] PE Hallett. The variations in visual threshold measurement. J Physiol, 202(2):403-419, 1969.

[22] HB Barlow and WR Levick. Changes in the maintained discharge with adaptation level in the cat retina. J Physiol, 202(3):699-718, 1969.

[23] WR Levick, LN Thibos, TE Cohn, D Catanzaro, and HB Barlow. Performance of cat retinal ganglion cells at low light levels. J Gen Physiol, 82(3):405-426, 1983.

[24] RG Smith, MA Freed, and P Sterling. Microcircuitry of the dark-adapted cat retina: functional architecture of the rod-cone network. J Neurosci, 6(12):3505-3517, 1986.

[25] AK Goodchild, KK Ghosh, and PR Martin. Comparison of photoreceptor spatial density and ganglion cell morphology in the retina of human, macaque monkey, cat, and the marmoset callithrix jacchus. J Comp Neurol, 366(1):55-75, 1996.

[26] BG Cleland, WR Levick, and H Wassle. Physiological identification of a morphological class of cat retinal ganglion cells. J Physiol, 248(1):151-171, 1975. 
[27] LJ Frishman and MW Levine. Statistics of the maintained discharge of cat retinal ganglion cells. J Physiol, 339:475-494, 1983.

[28] BE Saleh and MC Teich. Multiplication and refractoriness in the cat's retinal-ganglioncell discharge at low light levels. Biol Cybern, 52(2):101-107, 1985.

[29] F Rieke and D. A. Baylor. Single-photon detection by rod cells of the retina. Reviews of Modern Physics, 70:1027-1036, 1998.

[30] EN Jr Pugh and TD Lamb. Amplification and kinetics of the activation steps in phototransduction. Biochim Biophys Acta, 1141(2-3):111-149, 1993.

[31] TM Vuong, M Chabre, and L Stryer. Millisecond activation of transducin in the cyclic nucleotide cascade of vision. Nature, 311(5987):659-661, 1984.

[32] IB Leskov, VA Klenchin, JW Handy, GG Whitlock, VI Govardovskii, MD Bownds, TD Lamb, EN Jr Pugh, and VY Arshavsky. The gain of rod phototransduction: reconciliation of biochemical and electrophysiological measurements. Neuron, 27(3):525-537, 2000 .

[33] GD Field and F Rieke. Mechanisms regulating variability of the single photon responses of mammalian rod photoreceptors. Neuron, 35(4):733-747, 2002.

[34] GD Field and F Rieke. Nonlinear signal transfer from mouse rods to bipolar cells and implications for visual sensitivity. Neuron, 34(5):773-785, 2002.

[35] DA Baylor, G Matthews, and KW Yau. Two components of electrical dark noise in toad retinal rod outer segments. J Physiol, 309:591-621, 1980.

[36] F Rieke and DA Baylor. Molecular origin of continuous dark noise in rod photoreceptors. Biophys J, 71(5):2553-2572, 1996.

[37] F Rieke and DA Baylor. Origin of reproducibility in the responses of retinal rods to single photons. Biophys J, 75(4):1836-1857, 1998.

[38] GG Whitlock and TD Lamb. Variability in the time course of single photon responses from toad rods: termination of rhodopsin's activity. Neuron, 23(2):337-351, 1999.

[39] AC Aho, K Donner, C Hyden, LO Larsen, and T Reuter. Low retinal noise in animals with low body temperature allows high visual sensitivity. Nature, 334(6180):348-350, 1988.

[40] AC Aho, K Donner, and T Reuter. Retinal origins of the temperature effect on absolute visual sensitivity in frogs. J Physiol, 463:501-521, 1993.

[41] LT Sharpe and A Stockman. Rod pathways: the importance of seeing nothing. Trends Neurosci, 22(11):497-504, 1999.

[42] SA Bloomfield and RF Dacheux. Rod vision: pathways and processing in the mammalian retina. Prog Retin Eye Res, 20(3):351-384, 2001. 
[43] E Soucy, Y Wang, S Nirenberg, J Nathans, and M Meister. A novel signaling pathway from rod photoreceptors to ganglion cells in mammalian retina. Neuron, 21(3):481-493, 1998.

[44] MR Deans, B Volgyi, DA Goodenough, SA Bloomfield, and DL Paul. Connexin36 is essential for transmission of rod-mediated visual signals in the mammalian retina. Neuron, 36(4):703-712, 2002.

[45] B Volgyi, MR. Deans, DL Paul, and SA Bloomfield. Role of aii amacrine cell coupling in rod vision: Studies using a connexin36 knockout mouse. Invest. Ophthal. Vis. Sci. Suppl., S1944, 2002.

[46] H Kolb and R Nelson. Rod pathways in the retina of the cat. Vision Res, 23(4):301-312, 1983.

[47] RF Dacheux and E Raviola. The rod pathway in the rabbit retina: a depolarizing bipolar and amacrine cell. J Neurosci, 6(2):331-345, 1986.

[48] P Sterling, MA Freed, and RG Smith. Architecture of rod and cone circuits to the on-beta ganglion cell. J Neurosci, 8(2):623-642, 1988.

[49] R Nelson. Cat cones have rod input: a comparison of the response properties of cones and horizontal cell bodies in the retina of the cat. J Comp Neurol, 172(1):109-135, 1977.

[50] DM Schneeweis and JL Schnapf. Photovoltage of rods and cones in the macaque retina. Science, 268(5213):1053-1056, 1995.

[51] I Hack, L Peichl, and JH Brandstatter. An alternative pathway for rod signals in the rodent retina: rod photoreceptors, cone bipolar cells, and the localization of glutamate receptors. Proc Natl Acad Sci U S A, 96(24):14130-14135, 1999.

[52] Y Tsukamoto, K Morigiwa, M Ueda, and P Sterling. Microcircuits for night vision in mouse retina. J Neurosci, 21(21):8616-8623, 2001.

[53] Y Trifonov. Study of synaptic transmission between the photoreceptor and the horizontal cell using electrical stimulation of the retina. Biofizika, 13:809-817, 1968.

[54] JE Dowling and H Ripps. Effect of magnesium on horizontal cell activity in the skate retina. Nature, 242(5393):101-103, 1973.

[55] F Rieke and EA Schwartz. Asynchronous transmitter release: control of exocytosis and endocytosis at the salamander rod synapse. J Physiol, 493(Pt 1):1-8, 1996.

[56] J Del Castillo and B Katz. Statistical nature of facilitation at a single nerve-muscle junction. Nature, 171(4362):1016-1017, 1953.

[57] RR de Ruyter van Steveninck, GD Lewen, SP Strong, R Koberle, and W Bialek. Reproducibility and variability in neural spike trains. Science, 275(5307):1805-1808, 1997. 
[58] MJ 2nd Berry and M Meister. Refractoriness and neural precision. J Neurosci, 18(6):2200-2211, 1998.

[59] D Zenisek, JA Steyer, and W Almers. Transport, capture and exocytosis of single synaptic vesicles at active zones. Nature, 406(6798):849-854, 2000.

[60] D Attwell, S Borges, SM Wu, and M Wilson. Signal clipping by the rod output synapse. Nature, 328(6130):522-524, 1987.

[61] JH Belgum and DR Copenhagen. Synaptic transfer of rod signals to horizontal and bipolar cells in the retina of the toad (bufo marinus). J Physiol, 396:225-245, 1988.

[62] WB Thoreson, K Rabl, E Townes-Anderson, and R Heidelberger. A highly ca(2+)sensitive pool of vesicles contributes to linearity at the rod photoreceptor ribbon synapse. Neuron, 42(4):595-605, 2004.

[63] P Witkovsky, Y Schmitz, A Akopian, D Krizaj, and D Tranchina. Gain of rod to horizontal cell synaptic transfer: relation to glutamate release and a dihydropyridinesensitive calcium current. J Neurosci, 17(19):7297-7306, 1997.

[64] MC van Rossum and RG Smith. Noise removal at the rod synapse of mammalian retina. Vis Neurosci, 15(5):809-821, 1998.

[65] JG Robson and LJ Frishman. Response linearity and kinetics of the cat retina: the bipolar cell component of the dark-adapted electroretinogram. Vis Neurosci, 12(5):837$850,1995$.

[66] SM Saszik, JG Robson, and LJ Frishman. The scotopic threshold response of the darkadapted electroretinogram of the mouse. J Physiol, 543(Pt 3):899-916, 2002.

[67] DM Schneeweis and JL Schnapf. Noise and light adaptation in rods of the macaque monkey. Vis Neurosci, 17(5):659-666, 2000.

[68] CE Armstrong-Gold and F Rieke. Bandpass filtering at the rod to second-order cell synapse in salamander (ambystoma tigrinum) retina. J Neurosci, 23(9):3796-3806, 2003.

[69] AP Sampath and F Rieke. Selective transmission of single photon responses by saturation at the rod-to-rod bipolar synapse. Neuron, 41(3):431-443, 2004.

[70] S Nawy. The metabotropic receptor mglur6 may signal through g(o), but not phosphodiesterase, in retinal bipolar cells. J Neurosci, 19(8):2938-2944, 1999.

[71] EA Schwartz. Electrical properties of the rod syncytium in the retina of the turtle. $J$ Physiol, 257(2):379-406, 1976.

[72] DR Copenhagen and WG Owen. Coupling between rod photoreceptors in a vertebrate retina. Nature, 260(5546):57-59, 1976.

[73] PB Detwiler, AL Hodgkin, and PA McNaughton. Temporal and spatial characteristics of the voltage response of rods in the retina of the snapping turtle. J Physiol, 300:213-250, 1980 . 
[74] D Attwell, M Wilson, and SM Wu. A quantitative analysis of interactions between photoreceptors in the salamander (ambystoma) retina. J Physiol, 352:703-737, 1984.

[75] JL Schnapf and DR Copenhagen. Differences in the kinetics of rod and cone synaptic transmission. Nature, 296(5860):862-864, 1982.

[76] W Bialek and WG Owen. Temporal filtering in retinal bipolar cells. elements of an optimal computation? Biophys J, 58(5):1227-1233, 1990.

[77] F Rieke, WG Owen, and W Bialek. Optimal filtering in the salamander retina. Advances in Neural Information Processing Systems, Ed 3, pages 377-383, 1991.

[78] R Nelson and H Kolb. A17: a broad-field amacrine cell in the rod system of the cat retina. J Neurophysiol, 54(3):592-614, 1985.

[79] J Zhang, W Li, EB Trexler, and SC Massey. Confocal analysis of reciprocal feedback at rod bipolar terminals in the rabbit retina. J Neurosci, 22(24):10871-10882, 2002.

[80] E Hartveit. Reciprocal synaptic interactions between rod bipolar cells and amacrine cells in the rat retina. J Neurophysiol, 81(6):2923-2936, 1999.

[81] T Euler and RH Masland. Light-evoked responses of bipolar cells in a mammalian retina. J Neurophysiol, 83(4):1817-1829, 2000.

[82] CJ Dong and WA Hare. Temporal modulation of scotopic visual signals by a17 amacrine cells in mammalian retina in vivo. J Neurophysiol, 89(4):2159-2166, 2003.

[83] RG Smith and N Vardi. Simulation of the aii amacrine cell of mammalian retina: functional consequences of electrical coupling and regenerative membrane properties. Vis Neurosci, 12(5):851-860, 1995.

[84] N Vardi and RG Smith. The aii amacrine network: coupling can increase correlated activity. Vision Res, 36(23):3743-3757, 1996.

[85] SL Mills and SC Massey. Differential properties of two gap junctional pathways made by aii amacrine cells. Nature, 377(6551):734-737, 1995.

[86] DI Vaney. Many diverse types of retinal neurons show tracer coupling when injected with biocytin or neurobiotin. Neurosci Lett, 125(2):187-190, 1991.

[87] ML Veruki and E Hartveit. Electrical synapses mediate signal transmission in the rod pathway of the mammalian retina. J Neurosci, 22(24):10558-10566, 2002.

[88] SA Bloomfield, D Xin, and T Osborne. Light-induced modulation of coupling between aii amacrine cells in the rabbit retina. Vis Neurosci, 14(3):565-576, 1997.

[89] R Boos, H Schneider, and H Wassle. Voltage- and transmitter-gated currents of allamacrine cells in a slice preparation of the rat retina. J Neurosci, 13(7):2874-2888, 1993. 
[90] S Haverkamp, U Muller, K Harvey, RJ Harvey, H Betz, and H Wassle. Diversity of glycine receptors in the mouse retina: localization of the alpha3 subunit. $J$ Comp Neurol, 465(4):524-539, 2003.

[91] SH DeVries and DA Baylor. An alternative pathway for signal flow from rod photoreceptors to ganglion cells in mammalian retina. Proc Natl Acad Sci U S A, 92(23):1065810662, 1995.

[92] RH Masland and E Raviola. Confronting complexity: strategies for understanding the microcircuitry of the retina. Annu Rev Neurosci, 23:249-284, 2000.

[93] W Bialek. Physical limits to sensation and perception. Annu Rev Biophys Biophys Chem, 16:455-478, 1987.

[94] AM Angioy, A Desogus, IT Barbarossa, P Anderson, and BS Hansson. Extreme sensitivity in an olfactory system. Chem Senses, 28(4):279-284, 2003.

[95] AJ Kalmijn. Electric and magnetic field detection in elasmobranch fishes. Science, 218(4575):916-918, 1982. 